\title{
In Search of the SARS-CoV-2 Protection Correlate: Head-to-Head Comparison of Two Quantitative S1 Assays in Pre-characterized Oligo-/Asymptomatic Patients
}

Raquel Rubio-Acero $\cdot$ Noemi Castelletti $\cdot$ Volker Fingerle $\cdot$ Laura Olbrich $\cdot$ Abhishek Bakuli

Roman Wölfel · Philipp Girl · Katharina Müller · Simon Jochum · Matthias Strobl ·

Michael Hoelscher $\cdot$ Andreas Wieser on behalf of the KoCo19 study team

Received: March 25, 2021 / Accepted: May 28, 2021 / Published online: June 16, 2021

(c) The Author(s) 2021

\section{ABSTRACT}

Background: Quantitative serological assays detecting response to SARS-CoV-2 are needed to quantify immunity. This study analyzed the performance and correlation of two quantitative anti-S1 assays in oligo-/asymptomatic individuals from a population-based cohort.

Methods: In total, 362 plasma samples (108 with reverse transcription-polymerase chain

Raquel Rubio-Acero and Noemi Castelletti contributed equally/share first authorship.

The members of the KoCo19 study team are listed in the Acknowledgements.

Supplementary Information The online version contains supplementary material available at https://doi. org/10.1007/s40121-021-00475-x.

R. Rubio-Acero · N. Castelletti · L. Olbrich .

A. Bakuli · M. Hoelscher · A. Wieser ( $\square)$

Division of Infectious Diseases and Tropical

Medicine, University Hospital, Ludwig-Maximilians-

Universität (LMU) Munich, Leopoldstr. 5, 80802

Munich, Germany

e-mail: Wieser@mvp.lmu.de

N. Castelletti

Institute of Radiation Medicine, Helmholtz Zentrum

München, 85764 Neuherberg, Germany

V. Fingerle · L. Olbrich · R. Wölfel · P. Girl ·

K. Müller · M. Hoelscher · A. Wieser

German Center for Infection Research (DZIF),

Partner Site, Munich, Germany reaction [RT-PCR]-positive pharyngeal swabs, 111 negative controls, and 143 with positive serology without confirmation by RT-PCR) were tested with quantitative assays (Euroimmun Anti-SARS-CoV-2 QuantiVac enzyme-linked immunosorbent assay [EI-S1-IgG-quant]) and Roche Elecsys ${ }^{\circledR}$ Anti-SARS-CoV-2 S [Ro-RBD-Igquant]), which were compared with each other and confirmatory tests, including wild-type virus micro-neutralization (NT) and GenScript ${ }^{\circledR}$ CPass $^{\mathrm{TM}}$. Square roots $R$ of coefficients of determination were calculated for continuous variables and non-parametric tests were used for paired comparisons.

Results: Quantitative anti-S1 serology correlated well with each other (true positives, 96\%; true negatives, 97\%). Antibody titers decreased over time $(<30$ to $>240$ days after initial positive RT-PCR). Agreement with GenScript-cPass

\author{
V. Fingerle \\ Bavarian Health and Food Safety Authority (LGL), \\ Oberschleissheim, Germany

\section{Hoelscher} \\ Center for International Health (CIH), University \\ Hospital, LMU Munich, 80336 Munich, Germany \\ R. Wölfel · P. Girl · K. Müller \\ Bundeswehr Institute of Microbiology, 80937 \\ Munich, Germany \\ S. Jochum - M. Strobl \\ Roche Diagnostics GmbH, 82377 Penzberg, \\ Germany
}


was $96 \% / 99 \%$ for true positives and true negatives, respectively, for Ro-RBD-Ig-quant and 93\%/97\% for EI-S1-IgG-quant. Ro-RBD-Ig-quant allowed distinct separation between positives and negatives, and less non-specific reactivity versus EI-S1-IgG-quant. Raw values (95\% CI) $\geq 28.7 \mathrm{U} / \mathrm{mL}$ (22.6-36.4) for Ro-RBD-Igquant and $\geq 49.8 \mathrm{U} / \mathrm{mL}$ (43.4-57.1) for EI-S1IgG-quant predicted NT $>1: 5$ in $95 \%$ of cases.

Conclusions: Our findings suggest both quantitative anti-S1 assays (EI-S1-IgG-quant and RoRBD-Ig-quant) may replace direct neutralization assays in quantitative measurement of immune protection against SARS-CoV-2 in certain circumstances. However, although the mean antibody titers for both assays tended to decrease over time, a higher proportion of RoRBD-Ig-quant values remained positive after 240 days.

Keywords: COVID-19; Direct virus neutralization assay S1; Quantitative serology; SARS-CoV-2

\section{Key Summary points}

Quantitative serological assays detecting response to SARS-CoV-2 infection are urgently needed to quantify immunity.

This manuscript presents the results of direct comparison of two independent quantitative anti-S1 assays (Euroimmun Anti-SARS-CoV-2 QuantiVac ELISA [IgG] and Roche Elecsys ${ }^{\circledR}$ Anti-SARS-CoV-2 S) in oligo-/asymptomatic individuals from a previously characterized population-based cohort.

Both assays showed similar performance and a high level of agreement with direct virus neutralization and surrogate neutralization tests, arguing for their utility in quantifying immune protection against SARS-CoV-2.
In certain circumstances and following rigorous validation, these quantitative assays may replace direct neutralization assays (the current gold standard) which are unsuitable for large-scale studies and diagnostic routine testing.

\section{DIGITAL FEATURES}

This article is published with digital features, including a summary slide, to facilitate understanding of the article. To view digital features for this article go to https://doi.org/10.6084/ m9.figshare.14697141.

\section{INTRODUCTION}

Severe acute respiratory syndrome coronavirus 2 (SARS-CoV-2), the causative agent of coronavirus disease 2019 (COVID-19), emerged in Wuhan, China, in December 2019, and within months caused millions of infections and deaths across the globe [1]. Despite multiple interventions, including social distancing, wearing of protective equipment in public, and introducing enhanced disinfection procedures, the number of infected individuals worldwide continued to rise beyond the end of 2020 [2].

The gold standard for diagnosis of acute COVID-19 is molecular detection of the viral ribonucleic acid (RNA) by reverse transcriptionpolymerase chain reaction (RT-PCR) [3]. In addition, antibody testing can be used to detect humoral immune responses after the infection. Immunoassays detecting anti-SARS-CoV-2 antibodies can be especially valuable to confirm the extent of population exposure and to quantify vaccine responses $[4,5]$.

Seroconversion typically starts 5-7 days after SARS-CoV-2 infection [4]. All antibody types (immunoglobulin [Ig] A, IgG, and IgM) can be detected within the same time frame around week 2-4, and the IgG response persists the longest $[4,6]$. The most important targets of humoral response are the nucleocapsid protein $(\mathrm{N})$, involved in viral RNA replication and viral assembly, and parts of the trimeric spike 
complex, in particular the receptor-binding domain (RBD) of S1 which interacts with the angiotensin-converting enzyme 2 (ACE2) receptor on human cells [7, 8]. Antibodies that bind to RBD in a way that prevents its attachment to the host cell have a convincing functional likelihood to neutralize the virus and are viewed as the key indicator of immune protection [9-11]. In line with these observations, the spike protein became the leading antigen target in vaccine development [12].

Accumulating data suggest that high titers of IgG in convalescent plasma correlate with the presence of neutralizing antibodies, which may correlate with protection against infection $[8,13-15]$. However, the long-term persistence of neutralizing antibodies and the degree of protection they confer, as well as the degree and clinical significance of seroconversion in asymptomatic individuals, remain largely unknown.

A number of immunoassays from different manufacturers are currently available and have been compared directly in several head-to-head studies [16-19]. Since most are qualitative in nature, the emergence of quantitative assays is needed for a precise evaluation of immune response to viral antigens. Importantly, a reliable quantitative assay can be used to quantify protection in different settings (e.g., mild disease or vaccination). Such evaluation will require robust data on quantitative assay performance and its correlation with available neutralization tests.

Here, we present the results of a direct comparison of two novel quantitative anti-S1/RBD antibody tests applied to a subset of samples derived from a prospective population-based cohort study of COVID-19 incidence/prevalence in Munich, Germany. The ongoing KoCo19 study investigates the prevalence of SARS-CoV-2 infections among a randomly selected cohort, analyzes transmission within households and risk factors, and compares the performance of various immunoassays in testing asymptomatic and oligosymptomatic individuals [20]. The primary results were reported elsewhere including the estimated seroprevalance in Munich [21] and a comparison of seven qualitative seroassays to identify effective testing strategies [22]. This manuscript reports the analysis of the performance and correlation of quantitative Euroimmun Anti-SARS-CoV-2 QuantiVac enzyme-linked immunosorbent assay (ELISA) IgG assay that recognizes S1 (hereafter called EI-S1-IgG-quant) and quantitative Elecsys ${ }^{\circledR}$ Anti-SARS-CoV-2 S pan-Ig assay that recognizes S1 RBD (hereafter called RoRBD-Ig-quant) in a subset of samples derived from the KoCo19 cohort. Both assays were compared with previously described qualitative primary assays, namely Euroimmun Anti-SARSCoV-2 ELISA IgG (hereafter called EI-S1-IgG) $[23,24]$ and Elecsys Anti-SARS-CoV-2 Roche anti-N pan-Ig (hereafter called Ro-N-Ig) [25]. The primary tests were also assessed alongside assays that confirm infection, including direct virus neutralization test (NT) with SARS-CoV-2 wild-type virus (SARS-CoV-2 strain MUC-IMB01 isolated in January 2020), GenScript ${ }^{\circledR}$ CPass $^{\mathrm{TM}}$ (hereafter called GS-cPass) and Mikrogen-recomLine-N/RBD IgG line (hereafter called MG-N and MG-RBD) immunoassays.

\section{METHODS}

\section{Study Design and Participants}

Samples were derived from the populationbased prospective COVID-19 cohort KoCo19 from Munich, Germany [20]. Out of the total 6658 samples analyzed previously [22], 362 (due to having NT arrays and all other confirmatory tests) were included in this analysis. These included samples from (i) asymptomatic or oligosymptomatic individuals who had at least one RT-PCR-positive SARS-CoV-2 test on a pharyngeal swab (true positive samples), (ii) those who did not have an RT-PCR-positive test, but experienced seroconversion in at least one of the primary tests used ("other seropositive" samples), and (iii) negative controls-partially blood donors collected before the surge of SARSCoV-2/COVID-19, and negative samples obtained during the pandemic (true negative samples). All samples were collected during the same time period (between April and June 2020), except for true negative samples from blood donors (October 2019 and March 2020, 
i.e., before and after the seasonal common cold period). Samples were defined as "other seropositive" if one of the serological tests yielded a positive result, suggesting a likely but unconfirmed SARS-CoV-2 infection. To ensure a dataset with exclusively independent variables, only one serum sample per participant was used for analyses.

The study was approved by the Ethics Commission of the Faculty of Medicine at LudwigMaximilians-Universität Munich (reference numbers $20-275-\mathrm{V}, 20-371-\mathrm{V}$, and $20-262-\mathrm{V}$ ) and the protocol is available online (www. koco19.de) [20]. This study was conducted in accordance with the principles of the Declaration of Helsinki and Good Clinical Practice Guidelines. Informed written consent was obtained from all study participants.

\section{Laboratory Assays}

Blood samples were obtained as previously described [20]. Briefly, blood was collected in ethylenediaminetetraacetic acid-coated tubes, refrigerated and maintained at $4{ }^{\circ} \mathrm{C}$ from the moment of extraction until centrifugation to separate the cell pellet from the plasma. Plasma samples were analyzed and stored at $-80^{\circ} \mathrm{C}$ in temperature-controlled biobank freezers; freeze-thaw cycles were minimized to avoid sample degradation.

The presence of antibodies was analyzed using appropriate assay kits according to the manufacturers' instructions.

An overview of all assays, their cutoff values, and readouts are shown in Supplementary Table 1. The World Health Organization (WHO) reference sera (National Institute for Biological Standards and Control [NIBSC] code 20/268) were measured on the individual assays in replicates $(n=3)$ to standardize the results [26].

\section{Primary Assays}

EI-S1-IgG-quant and EI-S1-IgG results were measured on a Euroanalyzer-1 robot (Euroimmun, Lübeck, Germany). For the qualitative assay EI-S1-IgG, presented values show quotients of the optical density measurements given by the manufacturer's software. For the quantitative assay EI-S1-IgG-quant, values are shown in units per milliliter $(\mathrm{U} / \mathrm{mL})$. Two cutoffs were applied as recommended by the manufacturer: $25.6 \mathrm{U} / \mathrm{mL}$ to separate negative values from indeterminate values, and $35.2 \mathrm{U} /$ $\mathrm{mL}$ to separate indeterminate values from positive values (Supplementary Table 1). Values between 1 and $120 \mathrm{U} / \mathrm{mL}$ represent linear range, samples with values below $1 \mathrm{U} / \mathrm{mL}$ were assigned a categorical value of 0 , whereas samples with values above $120 \mathrm{U} / \mathrm{mL}$ were diluted 1:4 with sample buffer (Euroimmun, Lübeck, Germany) and measured again.

Ro-RBD-Ig-quant and Ro-N-Ig results were measured on cobas e411 and/or e801 modules (Roche, Mannheim, Germany). For the qualitative assay Ro-N-Ig, values correspond to the sample cutoff index. For the quantitative assay Ro-RBD-Ig, values are shown in U/mL. Manufacturer cutoff was applied to separate negative and positive values. Values between 0.4 and $250 \mathrm{U} / \mathrm{mL}$ represent linear range. Samples with values below $0.4 \mathrm{U} / \mathrm{mL}$ were assigned a categorical value of 0 , whereas samples with values above $250 \mathrm{U} / \mathrm{mL}$ were diluted 1:10 with sample diluent buffer (Roche, Mannheim, Germany).

\section{Assays Confirming Infection}

Confirmatory testing was performed using micro-virus NT assays as described previously [27], with the exception that confluent cells were incubated instead of adding cells following neutralization reaction, and the serum dilutions started with 1:5 instead of 1:10. Samples with a titer $<1: 5$ were classified as NT-negative, and samples with a titer $\geq 1: 5$ as NT-positive.

Binding inhibition was measured using the SARS-CoV-2 surrogate virus neutralization test (GS-cPass; GenScript ${ }^{\circledR}$, Piscataway, New Jersey, USA) according to the manufacturer's instructions. Photometric measurements were performed using the Tecan Sunrise (Tecan, Männedorf, Switzerland). Binding inhibition was calculated in percentages (range from $30 \%$ to $100 \%$; cutoff was $20 \%$ as recommended by the manufacturer).

The recomLine SARS-CoV-2 IgG line immunoassay (MG-N and MG-RBD; Mikrogen, Neuried, Germany) based on nitrocellulose strips with recombinant SARS-CoV-2 antigens $\mathrm{N}$ 
and RBD was measured using the fully automated recomLine strip processor CarL (Mikrogen, Neuried, Germany) according to the manufacturer's instructions. Raw values were presented in arbitrary units, and manufacturer's cutoffs were applied.

\section{Statistical Analysis}

Only one sample per participant was included in the statistical analyses; in case of individuals with multiple blood samples, the sample with the most comprehensive dataset was included. For multiple measurements with complete datasets, only the first measurement was considered; for operational replicates the latest measurement was included. Assay comparison was performed as described previously [22]. Statistical analysis and visualization was performed using software R, version 4.0.2 (https:// cloud.r-project.org/).

Square roots $R$ of coefficients of determination $\left(R^{2}\right)$ were calculated for continuous variables. Paired sample comparisons were performed using Wilcoxon-sign-rank tests; multiple group comparisons were performed using Kruskal-Wallis tests, followed by post hoc Dunn tests and the Benjamini-Yekutieli adjustment for pairwise comparisons [28].

\section{RESULTS}

A total of 362 samples from the KoCo19 cohort were included in the analysis: 108 samples from individuals who had viral RNA detected in pharyngeal swabs (true positives), 143 "other seropositive" samples, and 111 negative controls [20].

\section{Performance of Anti-S1 Tests}

The diagnostic accuracy measurements of all primary and confirmatory tests are presented in Table 1.

The raw value distributions of Ro-RBD-Igquant on $n=357$ evaluable samples and EI-S1IgG-quant on $n=354$ evaluable samples are presented in Fig. 1. Both assays showed a bimodal distribution. Ro-RBD-Ig-quant assay showed a better signal spread with a clear separation of true negative and true positive samples, and did not produce discordant results. RoRBD-Ig-quant detected $100 \%$ of the positive and $100 \%$ of the negative samples, whereas EI-S1IgG-quant detected $96 \%$ of the positive and $97 \%$ of the negative samples. Thirteen samples produced discordant results in EI-S1-IgG-quant and were categorized as indeterminate as they did not meet criteria for the positive or negative categories (Table 1).

Titer values of true positive samples with available data on time between RT-PCR and blood sampling for Ro-RBD-Ig-quant $(n=232)$ and EI-S1-IgG-quant $(n=228)$ are shown in Fig. 2. Values were widespread in the cohort with $<30$ days between RT-PCR and antibody test for both assays. The mean titer values tended to decrease over time, with statistically significant differences between value distribution in the cohort with $<30$ days vs cohort with $>240$ days for both assays $(p<0.0001)$. After 240 days, the majority (80\%) of Ro-RBDIg-quant values remained in the positive range whereas almost half of EI-S1-IgG-quant values no longer met the positivity threshold. Pairwise comparison between time-dependent groups after adjustment for multiple comparison are shown in Table 2.

\section{Concordance Between Quantitative and Semi-quantitative Anti-S1 Tests}

To allow for comparison of scale, results of individual assays with the WHO reference panel (NIBSC 20/168) are presented in Table 3.

Pairwise comparison of primary tests is shown in Supplementary Fig. 1; agreement of 95-98\% was observed for all comparisons. When categorical values were excluded, RoRBD-Ig-quant showed a high numerical correlation with EI-S1-IgG $(R=0.72, p<0.0001$; Supplementary Fig. 1A), while the numerical correlation with Ro-N-Ig was lower $(R=0.34$; $p<0.0001$; Supplementary Fig. 1B). EI-S1-IgGquant showed a high numerical correlation with EI-S1-IgG $(R=0.55, p<0.0001$; Supplementary Fig. 1C) while with Ro-N-Ig the 
Table 1 Performance of primary tests and confirmatory tests

\begin{tabular}{llllll}
\hline Test & $\boldsymbol{N}$ & $\begin{array}{l}\boldsymbol{n} \text { (true } \\
\text { positive) }\end{array}$ & $\begin{array}{l}\boldsymbol{n} \text { (true } \\
\text { negative) }\end{array}$ & $\begin{array}{l}\text { Positive result (\% of true } \\
\text { positive) }\end{array}$ & $\begin{array}{l}\text { Negative result (\% of true } \\
\text { negative) }\end{array}$ \\
\hline $\begin{array}{l}\text { EI-S1-IgG- } \\
\text { quant }\end{array}$ & 354 & 103 & 111 & $208(96)$ & $\begin{array}{l}133(97) \\
\text { Indeterminate: } 13(1)^{\mathrm{a}}\end{array}$ \\
$\begin{array}{l}\text { Ro-RBD-Ig- } \\
\text { quant }\end{array}$ & 357 & 107 & 111 & $202(100)$ & $155(100)$ \\
EI-S1-IgG & 362 & 108 & 111 & $232(98)$ & $130(98)$ \\
Ro-N-Ig & 361 & 108 & 111 & $201(98)$ & $160(98)$ \\
NT & 354 & 107 & 106 & $165(80)$ & $189(100)$ \\
GS-cPass & 360 & 108 & 111 & $198(96)$ & $162(99)$ \\
MG-N & 273 & 78 & 106 & $139(95)$ & $134(98)$ \\
MG-RBD & 273 & 78 & 106 & $137(95)$ & $136(100)$ \\
\hline
\end{tabular}

$R B D$ receptor-binding domain

a Thirteen samples (1\%) produced discordant results and were categorized as indeterminate

A

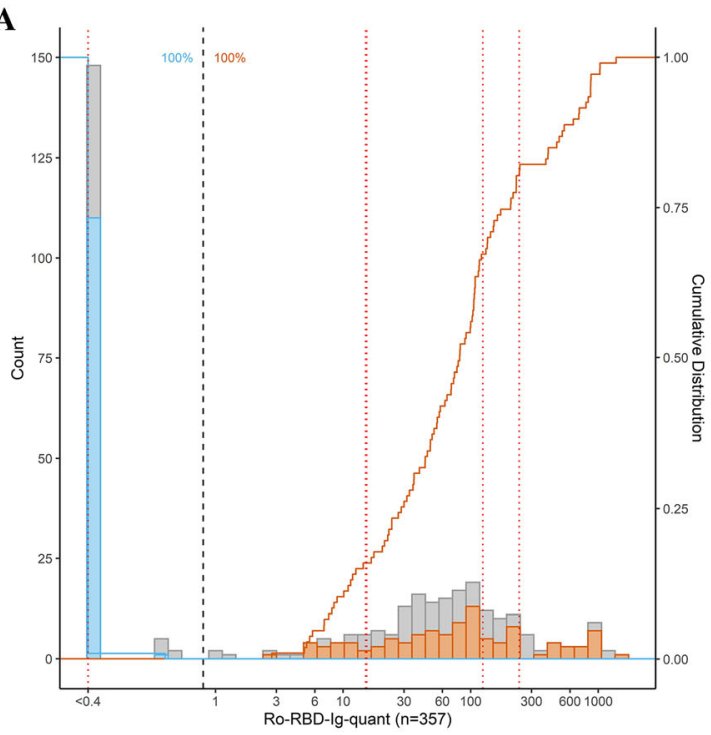

Fig. 1 Performance of primary tests Ro-RBD-Ig-quant (a) and EI-S1-IgG-quant (b). Black dashed lines represent manufacturers' cutoff values and red dotted lines represent WHO standards (from the left to the right: 20/142, $20 / 144$, and $20 / 140$ for $\mathbf{a}(20 / 140$ and $20 / 144$ for $\mathbf{b})$ with almost identical values, 20/148, 20/150). Histograms show

correlation was lower $(R=0.20, p<0.001$; Supplementary Fig. 1D). A high level of

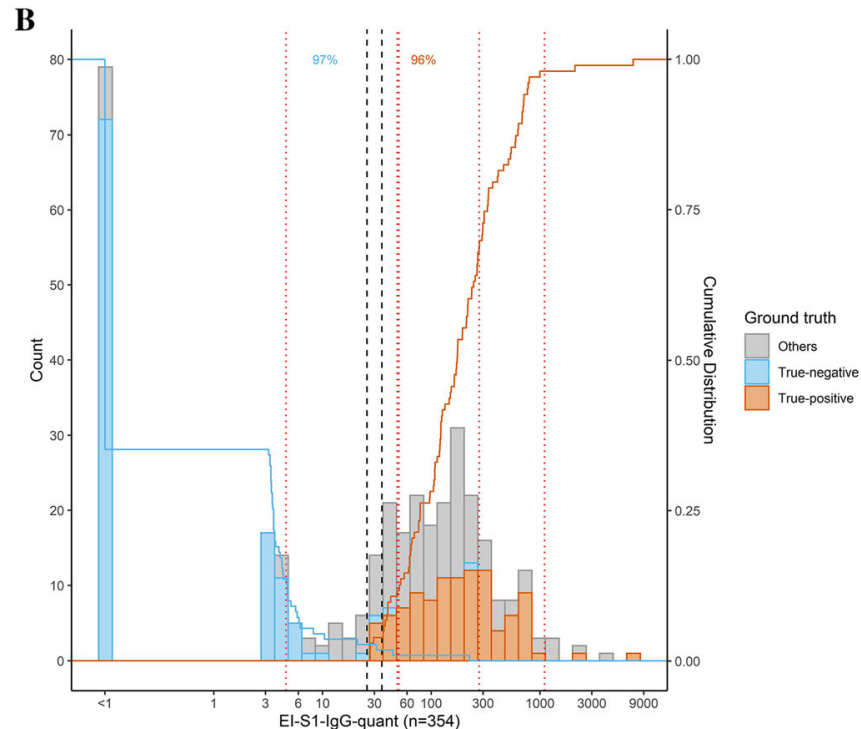

counts of individual samples, whereas solid blue and orange lines show cumulative distribution of true positive and true negative samples. Orange and blue numbers give the percentage of true positive and true negative samples, which were correctly detected by the tests

categorical agreement was observed between Ro-RBD-Ig-quant and EI-S1-IgG-quant with a 


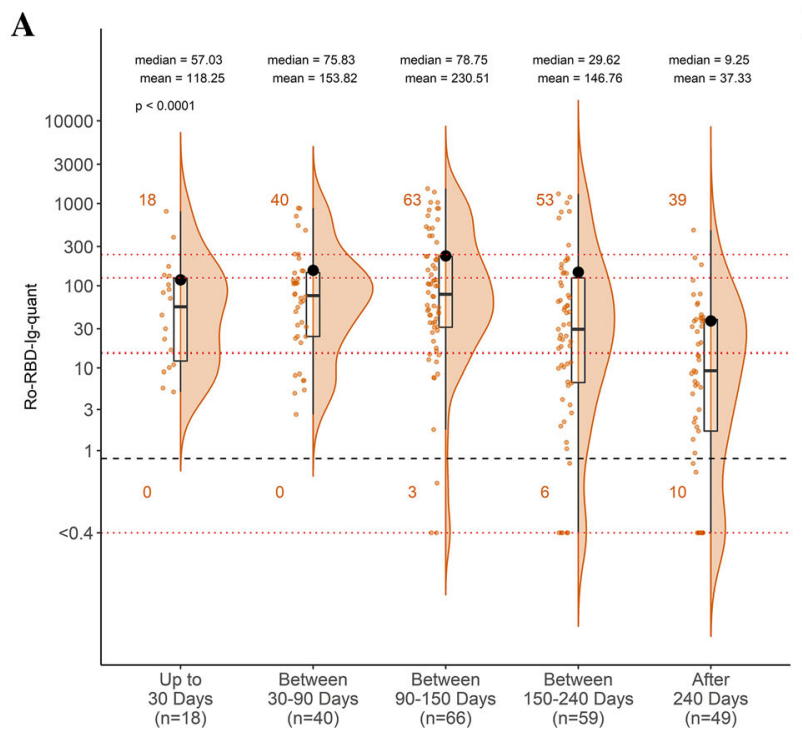

Fig. 2 Time-dependent values of positive samples in primary tests Ro-RBD-Ig-quant (a) and EI-S1-IgG-quant (b). Black dashed lines represent manufacturers' cutoff values and red dotted lines represent the WHO standards (from the bottom to the top: 20/142, 20/144, and 20/140 for a $(20 / 140$ and $20 / 144$ for b) with almost identical values, 20/148, 20/150). Assay results were categorized

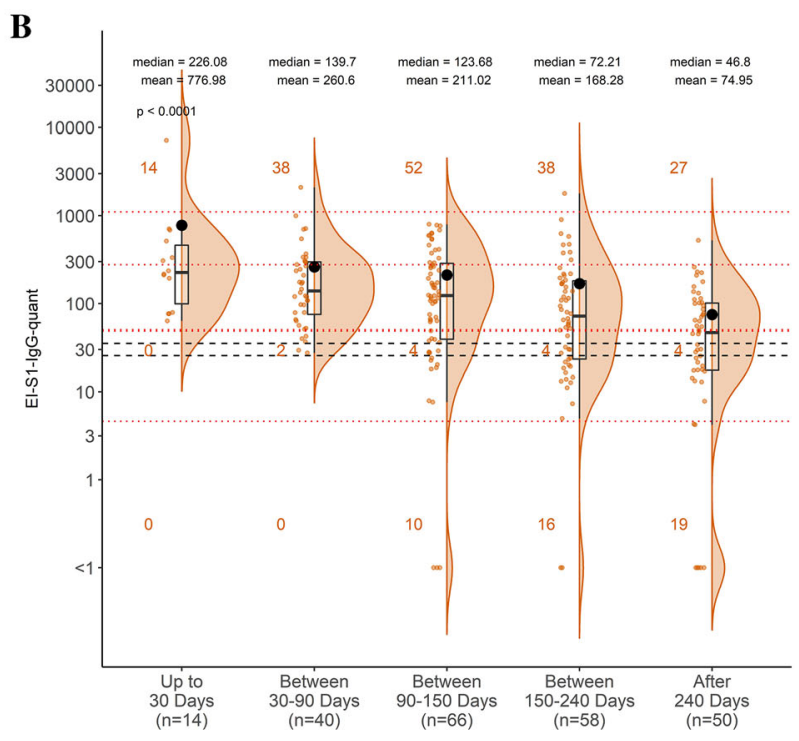

according to the time after the positive RT-PCR test (<30 days, 30-90 days, 90-150 days, 150-240 days, and $>240$ days). Plots show the individual read-out (orange dots), a density estimate (orange area), the 25th, 50th, and 75th percentiles (black box), and the mean (black dot); mean and median numbers are included for each group

Table 2 Pairwise comparison between the time-dependent groups after adjusting for multiple comparison

\begin{tabular}{lcr}
\hline Group comparison & \multicolumn{1}{c}{ Adjusted $p$ value } & EI-S1-IgG-quant \\
\cline { 2 - 3 } & Ro-RBD-Ig-quant & 0.71 \\
\hline Up to 30 days-between 30 and 90 days & 1.000 & 0.28 \\
Up to 30 days-between 90 and 150 days & 1.000 & $<0.05^{*}$ \\
Up to 30 days-between 150 and 240 days & 1.000 & $<0.001^{*}$ \\
Up to 30 days-after 240 days & $<0.05^{*}$ & 1.000 \\
Between 30 and 90 days-between 90 and 150 days & 1.000 & 0.169 \\
Between 30 and 90 days-between 150 and 240 days & $<0.001^{*}$ & $<0.001^{*}$ \\
Between 30 and 90 days-after 240 days & $<0.05^{*}$ & 0.16 \\
Between 90 and 150 days-between 150 and 240 days & $<0.0001^{*}$ & $<0.01^{*}$ \\
Between 90 and 150 days-after 240 days & $<0.05^{*}$ & 0.30 \\
Between 150 and 240 days-after 240 days & & \\
\hline
\end{tabular}

NISBC National Institute for Biological Standards and Contro, SD standard deviation

*Indicates the different levels of significance as shown by the $p$ values 
Table 3 Results from individual assays with the WHO reference panel for anti-SARS-CoV-2 immunoglobulin (NIBSC code 20/268)

\begin{tabular}{lllll}
\hline Reference level (NISBC code) & \multicolumn{4}{l}{ Mean titer value $(\boldsymbol{n}=\mathbf{3}),{ }^{\mathbf{a}}(\mathbf{S D})$} \\
\cline { 2 - 5 } & EI-S1-IgG-quant & Ro-RBD-Ig-quant & EI-S1-IgG & Ro-N-Ig \\
\hline Negative (20/142) & $4.62(0.27)$ & $<1(-)$ & $0.21(0.007)$ & $0.14(0.005)$ \\
Low (20/140) & $48.61(2.36)$ & $15.32(0.39)$ & $1.37(0.06)$ & $4.68(0.17)$ \\
Low anti-S, high anti-N (20/144) & $50.35(1.43)$ & $14.98(0.32)$ & $1.3(0.09)$ & $75.05(2.52)$ \\
Mid (20/148) & $276.62(10.45)$ & $124.47(2.49)$ & $4.33(0.12)$ & $101.89(1.00)$ \\
High (20/150) & $1103.25(4.95)$ & $239.87(2.75)$ & $6.56(0.13)$ & $118.29(3.16)$ \\
\hline
\end{tabular}

a Tests were repeated $\times 3$ and mean titer calculated

high correlation $(96 \%$ of positive samples and $97 \%$ of negative samples; $R=0.50, p<0.0001$; Supplementary Fig. 1E). Notably, Ro-RBD-Igquant gave a clearer separation of positive and negative values than EI-S1-IgG-quant; EI-S1IgG-quant showed many values at the intermediate range and some non-specific reactivity among the negative samples (3\%).

\section{Concordance with Tests Confirming Infection}

Ro-RBD-Ig-quant values showed significant increases between NT dilution categories $(p<0.001)$, with mean values increasing from 39.64 in the NT dilution category $<1: 5$ to 486.24 in the NT dilution category $>1: 80$ (Fig. 3a). Notably, NT at dilution 1:5 still contained approximately $20 \%$ of true positive samples. Ro-RBD-Ig-quant also showed a high categorical agreement and correlation with GScPass (96\%/99\%, $R=0.54, p<0.0001$; Fig. 3b).

EI-S1-IgG-quant values also showed significant increases between NT dilution categories, with mean values of EI-S1-IgG-quant increasing from 44.33 (NT dilution $<1: 5$ ) to 956.6 (NT dilution $>1$ :80; Fig. 3c). NT at dilution 1:5 still contained approximately $16 \%$ of true positive samples. EI-S1-IgG-quant showed a high level of correlation with GS-cPass $(93 \% / 97 \%, R=0.41$, $p<0.0001$; Fig. 3d), although some unspecific reactivity in the negative samples was detected for EI-S1-IgG (3\%).
The predictive value (95\% accordance of the positive predictive value) of the two quantitative tests at different thresholds was investigated through the alignment of their results with NT dilution categories $\geq 1: 5$ and $\geq 1: 10$, and GS-cPass categories $\geq 20 \%$ and $\geq 30 \%$. The lowest Ro-RBD-Ig-quant and EI-S1-IgG-quant values [with 95\% CI] for which GS-cPass is $\geq 20 \%$ (6.99 and 27.49 , respectively) or $\geq 30 \%$ (11.60 and 40.62) and NT is $\geq 1: 5$ (28.67 and 49.78 ) or $\geq 1: 10$ (51.41 and 104.06) are presented in Table 4 and Supplementary Fig. 2. These values refer to the intersection of the linear fit with the selected values for GS-cPass and NT of Fig. 3.

Ro-S1-Ig-quant showed a high level of correlation with line blot assay MG-RBD and a lower numerical correlation with MG-N $(R=0.44, p<0.0001$ and $R=0.32, p<0.001$, respectively; Supplementary Fig. 3A, B). EI-S1IgG-quant showed a high level of correlation with MG-RBD $(R=0.46, p<0.0001$; Supplementary Fig. 3C), but the agreement with MG-N was not statistically significant $(R=0.15$, $p=0.089$; Supplementary Fig. 3D).

\section{DISCUSSION}

Since the surge of the COVID-19 pandemic, the number of serological assays continues to increase, and the comparative assessment of their analytical performance is essential to inform strategies in diagnostic, epidemiological, 

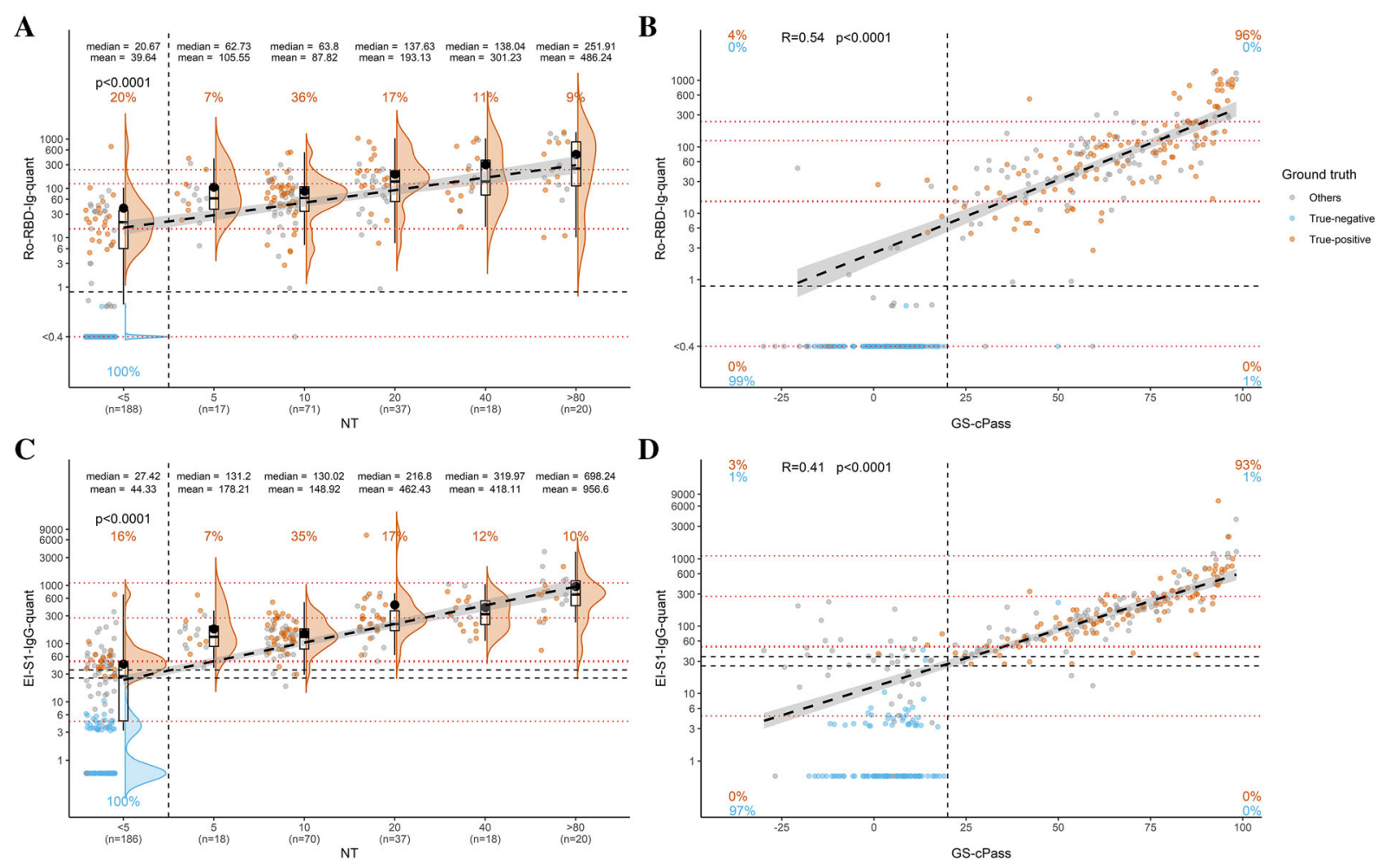

D

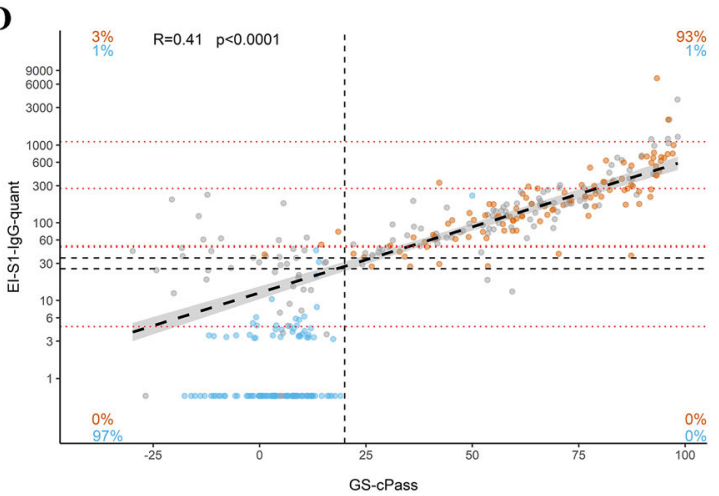

Fig. 3 Pairwise comparison of primary tests with confirmatory tests. Bivariate comparisons shown as violin and scatter plots for quantitative Ro-RBD-Ig-quant vs NT at indicated dilutions (a) and vs GS-cPass (b) and for quantitative EI-S1-IgG-quant vs NT at indicated dilutions (c) and vs GS-cPass (d). Black dashed lines represent manufacturers' cutoff values and red dotted lines represent the WHO standards (from the bottom to the top: 20/142, 20/144, and 20/140 for a (20/140 and 20/144 for c) with almost identical values, 20/148, 20/150). Orange and blue

numbers give the percentage of true positive and true negative samples, which were correctly detected by the tests. Bold dashed lines are linear fit and gray areas surrounding them represent 95\% CI; for the interested region, the polynomial fit was within the $95 \% \mathrm{CI}$ of the linear fit. Square root $R$ of coefficients of determination is given for association among continuous variables. Pairwise comparison between NT dilution categories (for a and $\mathbf{c}$ ) after adjustment for multiple comparison are shown in Table S2

and vaccination studies. In this study, we performed a head-to-head direct comparison of two independent quantitative assays directed against S1 and compared their performance with two qualitative primary assays and several assays that confirm infection, including direct virus neutralization. Samples from oligo-/ asymptomatic patients from a population-based cohort were utilized to help understand the true impact of COVID-19, given mild/asymptomatic cases are predominant in the population and data regarding immunity protection that develops in such patients are limited but of critical importance for policy makers worldwide.

The quantitative tests Ro-RBD-Ig-quant and EI-S1-IgG-quant showed a high level of correlation when used in a population cohort containing samples from mostly oligo- or asymptomatic individuals; both assays showed categorical agreement with Ro-N-Ig, micro-virus neutralization assay, GS-cPass and recomLine. This suggests both tests can detect correlates of neutralization, which is understood to mediate humoral protection following SARS-CoV-2 infection. While the mean titers for both assays tended to decrease after their peak (ca. 1 month or ca. 3 months after infection for EI-S1-IgGquant and Ro-RBD-Ig-quant, respectively) to $>240$ days after positive RT-PCR, a higher 
Table 4 Predictive value of quantitative tests

\begin{tabular}{lcc}
\hline NT/NT-surrogate & Quantitative serology (95\% CI) & \\
\cline { 2 - 3 } & Ro-S1-Ig-quant & EI-S1-IgG-quant \\
\hline GS-cPass $\geq 20$ & $\geq 6.99(5.30-9.21)$ & $\geq 27.49^{a}(23.94-31.57)$ \\
GS-cPass $\geq 30$ & $\geq 11.60(9.22-14.60)$ & $\geq 40.62(36.00-45.83)$ \\
NT $\geq 1: 5$ & $\geq 28.67(22.61-36.35)$ & $\geq 49.78(43.42-57.06)$ \\
NT $\geq 1: 10$ & $\geq 51.41(42.42-62.32)$ & $\geq 104.06(91.34-118.54)$ \\
\hline
\end{tabular}

Presented as lowest values (with $95 \%$ accordance of the positive predictive value $[95 \% \mathrm{CI}]$ ) of the quantitative tests for which NT is $\geq 1: 5$ and $\geq 1: 10$ and GS-cPass is $\geq 20 \%$ and $\geq 30 \%$

$C I$ confidence interval, $N T$ neutralization

${ }^{a}$ This value is below the positivity threshold

proportion of Ro-RBD-Ig-quant values remained positive after 240 days.

Finally, both quantitative assays showed a good level of concordance with each other, with Ro-RBD-Ig-quant performing slightly better in terms of clearer separation of positive and negative samples and less non-specific reactivity.

Currently, the most reliable method of detecting antibody response indicative of protection is direct virus neutralization; however, this test requires intact virus and has to be performed under biosafety level 3 conditions, making it infeasible for large-scale studies and diagnostic routine testing [17]. There are also numerous different protocols for direct viral neutralization with poor overall comparability. Commercial high-throughput tests use parts of viral proteins instead and combine these with other components of chemiluminescence detection or ELISA $[3,6]$. Although all viral proteins are likely to elicit some degree of immune response, most efforts concentrated on measuring antibodies directed against $\mathrm{N}$ and S1/ RBD so far [5]. Some studies suggest that $S 1$ may be the optimal antigen for SARS-CoV-2 serological tests, as it is more sensitive than RBD and more specific than S trimer [29]; however, this assumption could not be confirmed in this study.

Quantitative anti-S1 assays will be a valuable tool for measuring antibody responses to SARS$\mathrm{CoV}-2$. Importantly, quantitative assays will allow us to precisely enumerate and compare antibody titers in individuals who had severe disease, mild disease, asymptomatic individuals, and those who achieved immunity after vaccination. The assays may also be applied to screen for plasma samples that contain specific highaffinity neutralizing antibodies and help identify potential donors of plasma for convalescent plasma therapy [30]. Once established and rigorously validated, these assays may replace the current gold standard of direct neutralization, which requires handling at biosafety level 3 and has severe limitations in signal resolution at the lower end of the range.

In this study, both EI-S1-IgG-quant and RoRBD-Ig-quant showed a high level of correlation with direct virus micro-neutralization and surrogate neutralization test, GS-cPass. For example, raw values above $28.67 \mathrm{U} / \mathrm{mL}$ for Ro-RBDIg-quant and above $49.78 \mathrm{U} / \mathrm{mL}$ for EI-S1-IgGquant, respectively, predicted virus neutralization $>1: 5$ in $95 \%$ of cases. We may hypothesize that when the value of the quantitative tests is above the predictive value (e.g., 95\%), there is little benefit in performing NT and that this could act as a surrogate marker for neutralizing titers, e.g., after mass vaccinations or postinfection.

\section{CONCLUSIONS}

To the best of our knowledge, we present for the first time a comparison with quantitative tests which can be reproduced by others to assess 
neutralization as a bridge to serologically mediated immunoprotection. Our results suggest that both quantitative assays may be useful in future studies aimed to assess immunization efficiency, determine the degree of herd immunity, and estimate how long the response persists over time.

\section{ACKNOWLEDGEMENTS}

The authors thank all study participants for their trust, time, data, and samples, and all personnel at study centers and in the field for their contributions. ELECSYS is a trademark of Roche. All other product names and trademarks are the property of their respective owners. The members of KoCo19 investigators: Emad Alamoudi, Jared Anderson, Valeria Baldassare, Maximilian Baumann, Marieke Behlen, Marc Becker, Jessica Beyerl, Rebecca Böhnlein, Isabel Brand, Anna Brauer, Vera Britz, Jan Bruger, Friedrich Caroli, Lorenzo Contento, Alina Czwienzek, Flora Deák, Emma Dech, Laura Dech, Maximillian N Diefenbach, Jana Diekmannshemke, Anna Do, Gerhard Dobler, Ute Eberle, Juergen Durner, Ute Eberle, Judith Eckstein, Tabea Eser, Philine Falk, Jonathan Frese, Stefanie Fischer, Felix Forster, Turid Frahnow, Jonathan Frese, Günter Fröschl, Christiane Fuchs, Mercè Garí, Marius Gasser, Sonja Gauder, Otto Geisenberger, Christof Geldmacher, Kristina Gillig, Elias Golschan, Vitus Grauvogl, Jessica Michelle Guggenbuehl Noller, Celina Halfmann, Tim Haselwarter, Jan Hasenauer, Arlett Heiber, Matthias Herrmann, Stefan Hillmann, Christian Hinske, Janna Hoefflin, Tim Hofberger, Michael Höfinger, Larissa Hoffmann, Sacha Horn, Kristina Huber, Christian Janke, Ursula Kappl, Charlotte Kiani, Isabel Klugherz, Norah Kreider, Inge Kroidl, Arne Kroidl, Magdalena Lang, Clemens Lang, Silvan Lange, Ekaterina Lapteva, Michael Laxy, Ronan Le Gleut, Reiner Leidl, Felix Lindner, Alexander Maczka, Alicia Markgraf, Paula Matcau, Rebecca Mayrhofer, AnnaMaria Mekota, Hannah Müller, Dafni Metaxa, Leonie Pattard, Ivana Paunovic, Claire Pleimelding, Michel Pletschette, Michael Pritsch, Stephan Prückner, Kerstin Puchinger,
Konstantin Pusl, Peter Pütz, Katja Radon, Elba Raimúndez, Julius Raschka, Jakob Reich, Friedrich Riess, Camila Rothe, Elmar Saathoff, Nicole Schäfer, Yannik Schälte, Paul Schandelmaier, Lara Schneider, Sophie Schultz, Mirjam Schunk, Lars Schwettmann, Heidi Seibold, Peter Sothmann, Paul Stapor, Jeni Tang, Fabian Theis, Verena Thiel, Sophie Thiesbrummel, Eva Thumser, Niklas Thur, Julian Ullrich, Julia Waibel, Claudia Wallrauch, Simon Winter, Julia Wolff, Pia Wullinger, Tobias Würfel, Patrick Wustrow, Houda Yaqine, Sabine Zange, Eleftheria Zeggini, Thomas Zimmermann, Lea Zuche

Funding. This work was supported by Bavarian State Ministry of Science and the Arts; University Hospital; Ludwig-MaximiliansUniversität Munich; Helmholtz Centre Munich; University of Bonn; University of Bielefeld; German Ministry for Education and Research (proj. nr.: 01KI20271) and the Medical Biodefense Research Program of the Bundeswehr Medical Service. Euroimmun, Roche Diagnostics, Mikrogen, Viramed provided kits and machines for analyses at discounted rates. The Rapid Service and Open Access Fees were funded by Roche Diagnostics.

Medical Writing and/or Editorial Assistance. Editorial support was provided by Olga Ucar and Jade Drummond of inScience Communications, Springer Healthcare Ltd, UK, and was funded by Roche Diagnostics.

Authorship. All named authors meet the International Committee of Medical Journal Editors (ICMJE) criteria for authorship for this article, take responsibility for the integrity of the work as a whole, and have given their approval for this version to be published.

Authorship Contributions. Andreas Wieser, Michael Hoelscher, Raquel Rubio Acero designed the study, Raquel Rubio Acero, Volker Fingerle, Roman Wölfel, Philipp Girl, Katharina Müller performed laboratory analysis, Michael Hoelscher, Roman Wölfel, Laura Olbrich, Simon Jochum, Matthias Strobl, Raquel Rubio Acero and Andreas Wieser assisted with analysis of the data. Noemi Castelletti did data curation, 
data preparation and data cleaning, and together with Abhishek Bakuli, performed statistical analysis and figure generation. Laura Olbrich and Andreas Wieser provided samples and selected the patients from the KoCo19 study. Simon Jochum, Matthias Strobl, Noemi Castelletti, Raquel Rubio Acero, and Andreas Wieser prepared the manuscript. All authors have read and approved the final version of the manuscript.

Disclosures. Raquel Rubio Acero, Noemi Castelletti, Abhishek Bakuli, Roman Wölfel, Philipp Girl and Katharina Müller report no competing interests. Volker Fingerle reports honoraria and travel expenses for unrelated advanced trainings from Roche, Mikrogen, Diasorin; research grants from RKI, ECDC, INSTAND; Consultant for QCMD, INSTAND, Pfizer. Laura Olbrich reports non-financial support from Roche Diagnostics, Euroimmun, Viramed, Mikrogen, grants, non-financial support and other support from German Center for Infection Research (DZIF), grants and non-financial support from Government of Bavaria, non-financial support from BMW, non-financial support from Munich Police, non-financial support and other support from Accenture. Michael Hoelscher reports personal fees and non-financial support from Roche Diagnostics and DrBox, non-financial support from Euroimmun, Viramed and Mikrogen, grants, non-financial support and other support from German Center for Infection Research (DZIF), grants and non-financial support from Government of Bavaria, non-financial support from BMW, non-financial support from Munich Police, non-financial support and other support from Accenture. Simon Jochum and Matthias Strobl are employees of Roche Diagnostics $\mathrm{GmbH}$. Andreas Wieser reports personal fees and non-financial support from Roche Diagnostics and DrBox, non-financial support from Euroimmun, Viramed and Mikrogen, grants, non-financial support and other support from German Center for Infection Research (DZIF), grants and non-financial support from Government of Bavaria, non-financial support from BMW, non-financial support from Munich Police, non-financial support and other support from Accenture. Michael Hoelscher and Andreas Wieser report a pending patent application on a sample system for sputum diagnostics of SARS-CoV-2. Andreas Wieser is involved in other different patents and companies not in relation with the serology of SARSCoV-2. Andreas Wieser reports personal fees and other from Haeraeus Sensors, non-financial support from Bruker Daltonics, all of which are outside the submitted work, and non-related to SARS-CoV-2.

Compliance with Ethics Guidelines. The study was approved by the Ethics Commission of the Faculty of Medicine at Ludwig-Maximilians-Universität Munich (reference numbers $20-275-\mathrm{V}, 20-371-\mathrm{V}$, and $20-262-\mathrm{V})$. This study was conducted in accordance with the principles of the Declaration of Helsinki and Good Clinical Practice Guidelines. Informed written consent was obtained from all study participants.

Data Availability. Data are subject to data protection regulations and can be made available upon reasonable request to the corresponding author. To facilitate reproducibility and reuse, the code used to perform the analyses and generate the figures was made available on GitHub (https://github.com/koco19/lab_epi) and has been uploaded to ZENODO (http://doi. org/10.5281/zenodo.4300922, https://doi.org/ $10.5281 /$ zenodo.4300922) for long-term storage.

Open Access. This article is licensed under a Creative Commons Attribution-NonCommercial 4.0 International License, which permits any non-commercial use, sharing, adaptation, distribution and reproduction in any medium or format, as long as you give appropriate credit to the original author(s) and the source, provide a link to the Creative Commons licence, and indicate if changes were made. The images or other third party material in this article are included in the article's Creative Commons licence, unless indicated otherwise in a credit line to the material. If material is not included in the article's Creative Commons licence and your intended use is not permitted by statutory 
regulation or exceeds the permitted use, you will need to obtain permission directly from the copyright holder. To view a copy of this licence, visit http://creativecommons.org/licenses/by$\mathrm{nc} / 4.0 /$.

\section{REFERENCES}

1. Al-Rohaimi AH, Al OF. Novel SARS-CoV-2 outbreak and COVID19 disease; a systemic review on the global pandemic. Genes Dis. 2020;7(4):491-501.

2. European Centre for Disease Prevention and Control. COVID-19 country overviews. Week. 2020;51: 2020 .

3. Yan Y, Chang L, Wang L. Laboratory testing of SARS-CoV, MERS-CoV, and SARS-CoV-2 (2019$\mathrm{nCoV})$ : current status, challenges, and countermeasures. Rev Med Virol. 2020;30(3):e2106.

4. Carrillo J, Izquierdo-Useros N, Avila-Nieto C, Pradenas E, Clotet B, Blanco J. Humoral immune responses and neutralizing antibodies against SARSCoV-2; implications in pathogenesis and protective immunity. Biochem Biophys Res Commun. 2020;538:187-91.

5. Petherick A. Developing antibody tests for SARSCoV-2. Lancet. 2020;395(10230):1101-2.

6. Sethuraman $\mathrm{N}$, Jeremiah SS, Ryo A. Interpreting diagnostic tests for SARS-CoV-2. JAMA. 2020;323(22):2249-51.

7. Cheng MP, Yansouni CP, Basta NE, et al. Serodiagnostics for severe acute respiratory syndrome-related Coronavirus 2: a narrative review. Ann Intern Med. 2020;173(6):450-60.

8. Huang AT, Garcia-Carreras B, Hitchings MDT, et al. A systematic review of antibody mediated immunity to coronaviruses: kinetics, correlates of protection, and association with severity. Nat Commun. 2020;11(1):4704.

9. Piccoli L, Park YJ, Tortorici MA, et al. Mapping neutralizing and immunodominant sites on the SARS-CoV-2 spike receptor-binding domain by structure-guided high-resolution serology. Cell. 2020;183(4):1024-42 (e21).

10. Shi R, Shan C, Duan X, et al. A human neutralizing antibody targets the receptor-binding site of SARSCoV-2. Nature. 2020;584(7819):120-4.
11. Wang S, Peng Y, Wang R, et al. Characterization of neutralizing antibody with prophylactic and therapeutic efficacy against SARS-CoV-2 in rhesus monkeys. Nat Commun. 2020;11(1):5752.

12. Krammer F. SARS-CoV-2 vaccines in development. Nature. 2020;586(7830):516-27.

13. Brouwer PJM, Caniels TG, van der Straten K, et al. Potent neutralizing antibodies from COVID-19 patients define multiple targets of vulnerability. Science. 2020;369(6504):643-50.

14. Rogers TF, Zhao F, Huang D, et al. Isolation of potent SARS-CoV-2 neutralizing antibodies and protection from disease in a small animal model. Science. 2020;369(6506):956-63.

15. To KK-W, Tsang OT-Y, Leung W-S, et al. Temporal profiles of viral load in posterior oropharyngeal saliva samples and serum antibody responses during infection by SARS-CoV-2: an observational cohort study. Lancet Infect Dis. 2020;20(5):565-74.

16. Jaaskelainen AJ, Kuivanen S, Kekalainen E, et al. Performance of six SARS-CoV-2 immunoassays in comparison with microneutralisation. J Clin Virol. 2020;129:104512.

17. Kohmer N, Westhaus S, Ruhl C, Ciesek S, Rabenau HF. Brief clinical evaluation of six high-throughput SARS-CoV-2 IgG antibody assays. J Clin Virol. 2020;129:104480.

18. Weidner L, Gansdorfer S, Unterweger S, et al. Quantification of SARS-CoV-2 antibodies with eight commercially available immunoassays. J Clin Virol. 2020;129:104540.

19. National S-C-SAEG. Performance characteristics of five immunoassays for SARS-CoV-2: a head-to-head benchmark comparison. Lancet Infect Dis. 2020;20(12):1390-400.

20. Radon K, Saathoff E, Pritsch M, et al. Protocol of a population-based prospective COVID-19 cohort study Munich, Germany (KoCo19). BMC Public Health. 2020;20(1):1036.

21. Pritsch M, Radon K, Bakuli A, et al. Prevalence and risk factors of infection in the representative COVID-19 cohort Munich. Int J Environ Res Public Health. 2021;18(7):3572.

22. Olbrich L, Castelletti N, Schälte Y, et al. A serology strategy for epidemiological studies based on the comparison of the performance of seven different test systems: the representative COVID-19 cohort Munich. medRxiv. 2020. https://doi.org/10.1101/ 2021.01.13.21249735. 
23. EUROIMMUN anti-SARS-CoV-2 ELISA IgG, Package Insert. 2020.

24. Beavis KG, Matushek SM, Abeleda APF, et al. Evaluation of the EUROIMMUN Anti-SARS-CoV-2 ELISA Assay for detection of IgA and IgG antibodies. J Clin Virol. 2020;129:104468.

25. Muench P, Jochum S, Wenderoth V, et al. Development and Validation of the elecsys anti-SARSCoV-2 immunoassay as a highly specific tool for determining past exposure to SARS-CoV-2. J Clin Microbiol. 2020;58(10). https://doi.org/10.1128/ JCM.01694-20.

26. World Health Organization Expert Committee on Biological Standardization. WHO/BS.2020.2403 Establishment of the WHO International Standard and Reference Panel for anti-SARS-CoV-2 antibody, 9-10 December 2020. https://www.who.int/
publications/m/item/WHO-BS-2020.2403. Accessed 16 Feb 2021.

27. Haselmann V, Ozcurumez MK, Klawonn F, et al. Results of the first pilot external quality assessment (EQA) scheme for anti-SARS-CoV2-antibody testing. Clin Chem Lab Med. 2020;58(12):2121-30.

28. Yekutieli D, Benjamini Y. The control of the false discovery rate in multiple testing under dependency. Ann Stat. 2001;29(4):1165-88.

29. Tian Y, Lian C, Chen Y, et al. Sensitivity and specificity of SARS-CoV-2 S1 subunit in COVID-19 serology assays. Cell Discov. 2020;6:75.

30. Ouyang J, Isnard S, Lin J, et al. Convalescent plasma: the relay baton in the race for coronavirus disease 2019 treatment. Front Immunol. 2020;11: 570063. 